\title{
Early Experiences of a Minimal Invasive Intra-Prostatic Implant, Prostatic Urethral Lift for Benign Prostatic Hyperplasia Treatment in Korea
}

\author{
Jonghyup Yang, Dongho Shin, Jaewoo Sung, Shinjay Cho, Sungmin Kang, Hyun Cheol Jeong ${ }^{1}$, Sae Woong Choi ${ }^{2}$, \\ Woong Jin Bae, Sae Woong Kim
}

Department of Urology, The Catholic University of Korea, Seoul St. Mary's Hospital, College of Medicine, The Catholic University of Korea, Seoul, ${ }^{1}$ Department of Urology, Kangdong Sacred Heart Hospital, Seoul, ${ }^{2}$ Department of Urology, The Catholic University of Korea, Yeouido St. Mary's Hospital, College of Medicine, The Catholic University of Korea, Seoul, Korea

Purpose: As a minimal invasive device for benign prostatic hyperplasia (BPH) treatment, prostatic urethral lift (PUL) is widely accepted worldwide but not widely used in Korea. We investigated the one-year results of for patients with $\mathrm{BPH}$ in Korea. Materials and Methods: From April 2017 to June 2018, 42 patients with BPH were treated with PUL under local anesthesia with sedation. International Prostate Symptom Score (IPSS) and maximum urinary flow rate and post-void residual (PVR) were evaluated preoperatively and 1, 3, 6, and 12 months later.

Results: Mean age was $69.57 \pm 8.58$ years old, and mean prostatic volume was $37.17 \pm 12.19$ cc. Preoperative total IPSS and quality of life (QOL) were $19.94 \pm 7.81$ and $3.69 \pm 1.30$, respectively. Total IPSS improved to 11.26 \pm 7.22 $(p<0.001)$, and QOL was 2.42 $\pm 1.43(p=0.01)$ after one month. Patients showed no evidence of inflammation related to the implants. IPSS and QOL were somewhat worse after 3 months but were better than baseline at 6 and 12 months. Preoperative maximum flow rate (Qmax) was $9.71 \pm 5.45 \mathrm{ml} / \mathrm{sec}$, and one month after surgery, it had improved to $12.63 \pm 7.33(p=0.01)$; it remained good at 3,6 , and 12 months $(12.63 \pm 7.38,12.45 \pm 7.39,14.73 \pm 9.67)$. PVR was not significant at any points postoperative $(80.61 \pm 67.91$ to $43.95 \pm 8.19, p=0.119)$. No patient reported retrograde ejaculation, erectile dysfunction or urinary tract infection.

Conclusions: We evaluated the one-year efficacy of PUL for BPH treatment in Korea, and found significant improvement of IPSS, QOL and Qmax. It is expected that not only the improvement of voiding symptom but also the preservation of sexual function with a low risk of adverse events.

Keywords: Prostatic hyperplasia; Lower urinary tract symptoms; Transurethral resection of prostate; Adrenergic alpha-antagonists

Copyright (c) 2019, Korean Association of Urogenital Tract Infection and Inflammation. All rights reserved. (c) (1) (\$) This is an open access article distributed under the terms of the Creative Commons Attribution unrestricted non-commercial use, distribution, and reproduction in any medium, provided the original work is properly cited.
Received: 30 May, 2019

Revised: 4 September, 2019

Accepted: 9 October, 2019
Correspondence to: Sae Woong Kim
(i) https://orcid.org/0000-0002-9127-9400
Department of Urology, The Catholic University of
Korea, Seoul St. Mary's Hospital, College of Medi-
cine, The Catholic University of Korea, 222 Banpo-
daero, Seocho-gu, Seoul 06591, Korea
Tel: +82-2-2258-1401, Fax: +82-2-2258-1536
E-mail: ksw1227@catholic.ac.kr

\section{INTRODUCTION}

As a part of senile changes, the prostate gland enlarges, which causes a variety of male lower urinary tract symptoms (LUTS). According to the United States data, approximately
$30 \%$ of men over the age of 50 years suffer from benign prostatic hyperplasia (BPH) [1,2]. In Korea, the prevalence of $\mathrm{BPH}$ was $36 \%, 43 \%$, and $53 \%$ in the 1960 s, 1970 s, and 1980s, respectively, indicating that many patients suffer from $\mathrm{BPH}$ [3]. Medical treatments, such as alpha blockers and 
5-alpha reductase inhibitors, are effective for BPH-related LUTS but have many side effects, such as orthostatic hypotension, asthenia, dizziness, headaches, decreased libido, and erectile dysfunction [4].

A transurethral resection of the prostate (TURP) is still considered the gold standard treatment for BPH-related LUTS offering the best International Prostate Symptom Score (IPSS) improvement [4]. On the other hand, TURP has approximately $20 \%$ perioperative morbidity and long-term complications, including urinary incontinence (3\%), stricture $(7 \%)$, and erectile (10\%), and ejaculatory dysfunction (65\%) [5,6]. In addition, a long hospital stay and urethral catheter indwelling are required and there are some risks of severe hematuria.

Recently, several techniques for minimally invasive surgery have been developed and used to treat BPH. Transurethral microwave therapy, transurethral needle ablation of the prostate, and prostatic urethral lift (PUL) are all minimally invasive surgeries that can be performed under local anesthesia and have similar effectiveness and less morbidity than TURP [7-9]. Unlike conventional surgery, PUL is a technique that compresses both lateral lobes of the prostate using a permanent implant that does not cause any thermal injury to the surrounding tissues. Therefore, there is less hemorrhage with PUL and it can be performed in an outpatient setting. In addition, it has the advantage of preserving the ejaculatory function [10-12]. This paper reports for the first time in Korea the authors' experiences with the one-year use of the PUL system in BPH-related LUTS patients.

\section{MATERIALS AND METHODS}

In this study, data on 42 patients, who underwent PUL from April 2017 to June 2018, were collected retrospectively. The patients had been diagnosed with symptomatic BPH and had the desired surgical management with a previous alpha-blocker treatment (Table 1). A Foley urethral catheter was placed after surgery and removed on the same day. Whenever possible, the patients were scheduled to be discharged on the same day, but one patient was admitted to hospital for personal reasons.

All patients underwent preoperative evaluations, including a urodynamic study and transrectal ultrasound (TRUS) to measure the prostate size and cystoscopy to check if the
Table 1. Subjects' demographics

\begin{tabular}{lc}
\hline \multicolumn{1}{c}{ Demographics } & Value \\
\hline Age $(\mathrm{y})$ & $69.57 \pm 8.58$ \\
Number of anchors & $2.24(2-4)$ \\
Prostate volume $(\mathrm{cc})$ & $37.17 \pm 12.19$ \\
Prostate specific antigen $(\mathrm{ng} / \mathrm{ml})$ & $2.11 \pm 2.10$ \\
Total IPSS & $19.94 \pm 7.81$ \\
IPSS voiding & $12.06 \pm 5.67$ \\
IPSS storage & $7.11 \pm 3.42$ \\
IPSS QOL & $3.69 \pm 1.30$ \\
Qmax $(\mathrm{ml} / \mathrm{sec})$ & $9.71 \pm 5.45$ \\
PdetQmax $\left(\mathrm{cm} / \mathrm{H}_{2} \mathrm{O}\right)$ & $52.34 \pm 29.53$ \\
BOOI & $37.04 \pm 29.73$ \\
BCl & $90.59 \pm 35.55$ \\
Schäfer grade & $2.58 \pm 1.30$ \\
PVR (ml) & $80.61 \pm 67.91$ \\
\hline
\end{tabular}

Values are presented as mean \pm standard deviation or average (range). IPSS: International Prostate Symptom Score, QOL: quality of life, Qmax: maximum flow rate, PdetQmax: detrusor pressure at peak flow rate, BOOI: bladder outlet obstruction index, $\mathrm{BCI}$ : bladder contractility index, PVR: post-void residual.

median lobe of the prostate could tolerate the procedure. Patients were excluded if they had a prostate larger than $80 \mathrm{~cm}^{3}$ or median lobe hypertrophy, serum prostate-specific antigen level higher than $10 \mathrm{ng} / \mathrm{ml}$, a history of urinary retention or prostate surgery, renal insufficiency, or current infection.

Follow-up visits were conducted at one week and at three, six, and 12 months. The IPSS, quality of life (QOL) score, maximum flow rate (Qmax), and post-void residual (PVR) were used to assess the LUTS relief and functional improvement. The IPSS questionnaire was administered to determine the study eligibility and again after enrollment to provide a baseline value for analysis. The IPSS results for the earliest treated patients are also reported as indicators of the long-term effectiveness. The safety was assessed by the severity of the device or procedure-related adverse events using the Clavien-Dindo classification. The IPSS, maximum urinary flow rate, and PVR urine were also assessed preoperatively and one, three, six, and 12 months later. The Institutional Review Board of The Catholic University of Korea approved this study (approval number: KC18RESI0747). All subjects provided informed consent when they were enrolled.

\section{Procedure}

The transurethral PUL was performed with the patient under local anesthesia. Local anesthesia was typically coupled with light sedation and achieved through either the urethral 
instillation of lidocaine jelly or lidocaine bladder instillation mixed with normal saline. After performing rigid cystoscopy, the implant delivery device (UroLift ${ }^{\circledR}$ System; NeoTract, Pleasanton, CA, USA) was inserted into the 20-Fr sheath. This device compresses a prostatic lateral lobe in the anterolateral direction toward the capsule and advances a spring-activated, 19-gauge, 33-mm needle through the prostatic lobe and capsule (Fig. 1). The needle, housing a monofilament with a metallic tab, was then deployed through the prostate lobe. As the needle was retracted, the tab engaged the prostate capsule, and the monofilament was tensioned. Finally, the urethral end-piece was attached to the monofilament, which was then cut, delivering the in situ sized implant. Because the fibromuscular capsule was less compliant than the periurethral tissue, the capsular tab was held firmly in place while the urethral end-piece held the lobe in its displaced position, thereby expanding the urethral lumen. When implanted, the urethral end-piece invaginated into the urethral wall, where a focal injury promoted epithelialization. The objective of the PUL was to form a channel through the anterior aspect of the prostatic fossa.

\section{Statistical Analysis}

A paired t-test and Wilcoxon signed rank test was performed for continuous variables to compare clinical
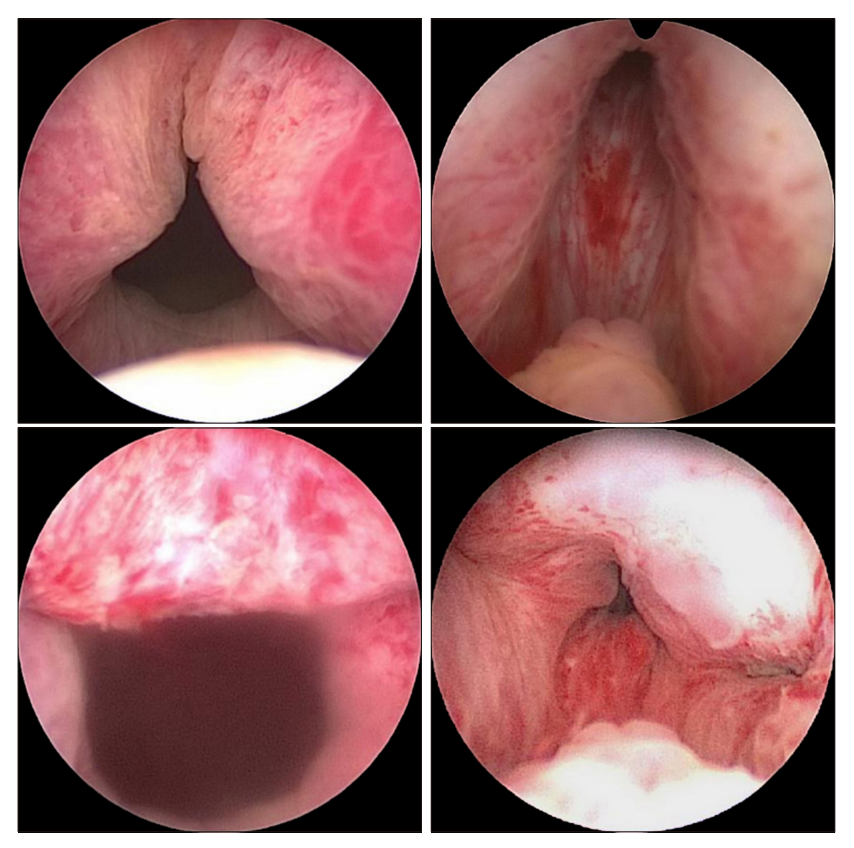

Fig. 1. Cystoscopic features before (upper) and after (lower) prostatic urethral lift patients. differences from the baseline, and differences were considered statistically significant at $\mathrm{p}<0.05$. All $\mathrm{p}$-values reported were two sided. The analyses were performed using IBM SPSS Statistics ver. 22.0 (IBM Corp., Armonk, NY, USA).

\section{RESULTS}

Forty-two patients underwent the procedure, and all procedures were completed successfully. The mean age and prostatic volume were $69.57 \pm 8.58$ years old and $37.17 \pm$ $12.19 \mathrm{cc}$, respectively. The patients received an average of 2.24 (2-4) implants. The mean operation time was $17.5 \pm 3.54$ minutes. The mean detrusor pressure at peak flow rate (PdetQmax) was $52.34 \pm 29.53 \mathrm{~cm} / \mathrm{H}_{2} \mathrm{O}$ and the Schäfer grade was $2.58 \pm 1.30$. The mean bladder outlet obstruction index (BOOI) and mean bladder contractility index (BCI) was $37.04 \pm 29.73$ and $90.59 \pm 35.55$, respectively.

PUL improved LUTS rapidly and significantly throughout the one-year follow-up period (Table 2). The preoperative

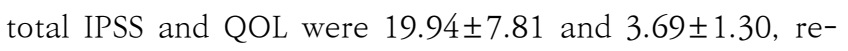
spectively. The total IPSS improved significantly to $11.26 \pm$ $7.22(\mathrm{p}<0.001)$ and the QOL was $2.42 \pm 1.43(\mathrm{p}=0.01)$ after one month; the IPSS and QOL were worse after three months (11.74 \pm 7.08 and $2.22 \pm 1.37$, respectively) but remained better than the baseline at six and 12 months. All symptomatic improvement was significantly different after six months, but some parameters were not significant at 12 months. PUL was effective in improving the voiding function, as assessed by the Qmax; the Qmax improved significantly after six months $(\mathrm{p}=0.03)$ and by $52 \%$ at one year, but this was no longer statistically significant. The PVR displayed a large variance but remained stable from the baseline to one year. The data after 12 months showed improvement in many parameters but were not statistically significant. The decrease in the number of patients at 12 months may explain this (Fig. 2).

The early postoperative course was typical of an endoscopic procedure with irritative symptoms, urinary retention, dysuria, and mild hematuria, which are typically resolved within the first week (all Clavien-Dindo grade 1, Table 3). One patient required catheterization due to gross hematuria, but the symptoms improved after irrigation and catheter removal. No patient required a blood transfusion, and none developed stress incontinence. Three patients required catheterization for urinary retention after the procedure. In two 
Table 2. Change in the outcomes after prostatic urethral lift: one month to 12 months

\begin{tabular}{cccccc}
\hline Parameter & Baseline $(\mathrm{n}=42)$ & 1 month $(\mathrm{n}=42)$ & 3 months $(\mathrm{n}=42)$ & 6 months $(\mathrm{n}=25)$ & 12 months $(\mathrm{n}=5)$ \\
\hline IPSS & $19.94 \pm 7.81$ & $11.26 \pm 7.22$ & $11.74 \pm 7.08$ & $15.38 \pm 6.79$ & $13.33 \pm 9.02$ \\
p-value & & $<0.001$ & $<0.001$ & 0.001 & 0.18 \\
IPSS voiding & $12.06 \pm 5.66$ & $5.71 \pm 5.17$ & $6.67 \pm 5.34$ & $8.62 \pm 4.48$ & $7 \pm 6$ \\
p-value & & $<0.001$ & 0.002 & 0.02 & 0.126 \\
IPSS storage & $7.11 \pm 3.42$ & $5.55 \pm 2.64$ & $5.07 \pm 2.43$ & $6.77 \pm 3.00$ & $6.33 \pm 3.06$ \\
p-value & & 0.004 & 0.001 & 0.03 & 0.057 \\
IPSS QOL & $3.69 \pm 1.30$ & $2.42 \pm 1.43$ & $2.22 \pm 1.37$ & $2.77 \pm 1.48$ & $3 \pm 1$ \\
p-value & & 0.01 & 0.64 & 0.636 & 0.573 \\
Qmax (ml/sec) & $9.71 \pm 5.45$ & $12.63 \pm 7.33$ & $12.63 \pm 7.38$ & $12.45 \pm 7.39$ & $14.73 \pm 9.67$ \\
p-value & & 0.01 & 0.002 & 0.03 & 0.324 \\
PVR (ml) & $80.61 \pm 67.91$ & $43.95 \pm 8.19$ & $33.23 \pm 48.81$ & $31.65 \pm 39.71$ & $19.33 \pm 17.01$ \\
p-value & & 0.119 & 0.003 & 0.206 & 0.5 \\
\hline
\end{tabular}

Values are presented as mean \pm standard deviation.

IPSS: International Prostate Symptom Score, QOL: quality of life, Qmax: maximum flow rate, PVR: post-void residual. p-value compares clinical differences from baseline.

A
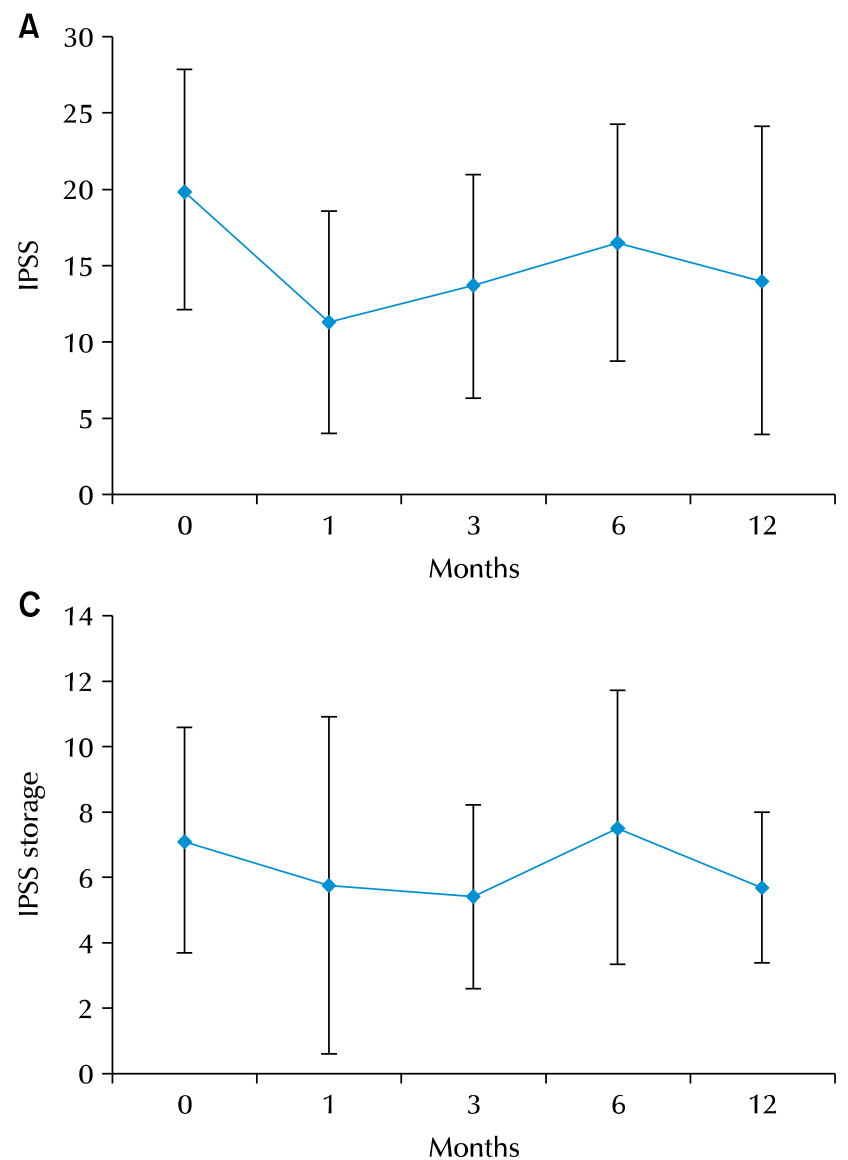
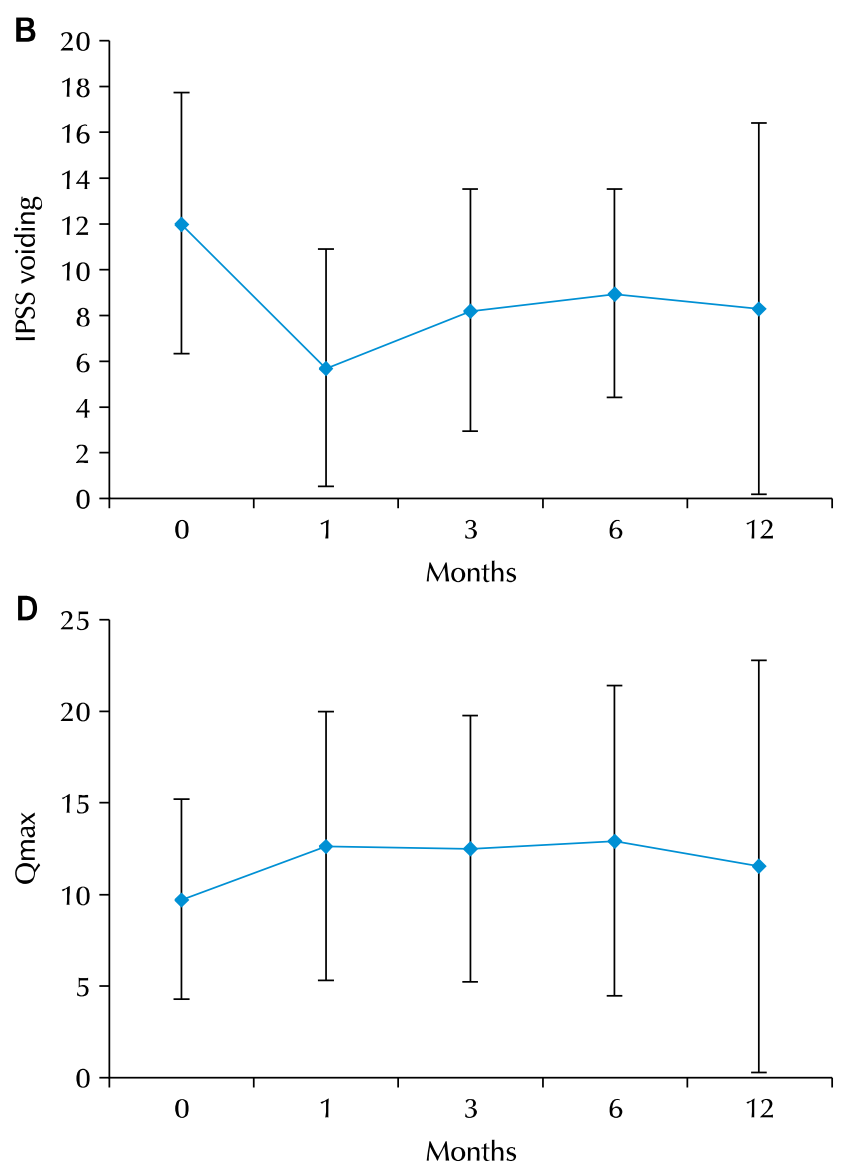

Fig. 2. Preoperative, one, three, six, and 12 months results for prostatic urethral lift. (A) Total International Prostate Symptom Score (IPSS), (B) IPSS voiding score, (C) IPSS storage score, (D) maximum flow rate (Qmax).

patients, the urethral catheter could be removed after one week, but a third had to keep the catheter for more than one month. No sexual dysfunction, such as erectile dysfunction, retrograde ejaculation was reported after PUL. No patients had Clavien-Dindo grade 2 symptoms.

\section{DISCUSSION}

Assuming that $30 \%$ of men 50 years or older will eventually suffer from BPH-related LUTS, the number is expected to be around four million patients in South Korea. On the other 
Table 3. Overview of adverse events over one year after the prostatic urethral lift

\begin{tabular}{lcccc}
\hline \multicolumn{1}{c}{ Adverse events } & $\begin{array}{c}0-1 \\
\text { month }\end{array}$ & $\begin{array}{c}1-3 \\
\text { months }\end{array}$ & $\begin{array}{c}3-6 \\
\text { months }\end{array}$ & $\begin{array}{c}6-12 \\
\text { months }\end{array}$ \\
\hline $\begin{array}{l}\text { Total available subjects } \\
\text { Related adverse events }\end{array}$ & 42 & 32 & 18 & 5 \\
Clavien grade 1 & 5 & 1 & 0 & 0 \\
Dysuria & $1(2.4)$ & 0 & 0 & 0 \\
Urinary retention & $3(7.1)$ & $1(3.1)$ & 0 & 0 \\
Hematuria & $1(2.4)$ & 0 & 0 & 0 \\
Urinary tract infection & 0 & 0 & 0 & 0 \\
Erectile dysfunction & 0 & 0 & 0 & 0 \\
Retrograde ejaculation & 0 & 0 & 0 & 0 \\
Clavien grade 2 & 0 & 0 & 0 & 0 \\
\hline
\end{tabular}

Values are presented as number only or number (\%).

hand, according to 2014 reports, only approximately 100,000 Korean men visited a hospital due to BPH [13]. Among these, only $2.1 \%$ received surgical treatments [14]. Patients decide against surgery for many reasons, such as morbidity concerns, long hospital stays, and anxiety about erectile and ejaculatory dysfunction in younger patients.

The prostate enlarges daily as men age until they die [15], but a decreased bladder function is difficult to recover. Therefore, it is important to start $\mathrm{BPH}$ treatment at an appropriate time to preserve the bladder function. More men than previously thought, particularly men under 50 years of age and those with a bladder outlet obstruction (BOO), value preserving the ejaculatory function [16]. Therefore, patients who require early $\mathrm{BOO}$ treatment to preserve the bladder function often hesitate because of concerns about the ejaculatory and sexual function. In these cases, PUL may be a good treatment alternative.

In United States, the total cost of PUL with two implants is similar to the cost of TURP; PUL with urodynamic study, cystoscopy, and TRUS using four implants of PUL costs approximately US $\$ 6,230$ in the United States, and the total for TURP with a diagnostic evaluation is approximately US $\$ 4,821$ [17]. In Korea, however, the cost of TURP is approximately US $\$ 650$, which is less than the price of just one PUL implant [13]. Therefore, if PUL requires two or more implants, the patient's cost is more than double that for TURP. Because of the high prostate volume and the burden of the patient due to the high cost of surgery, PUL was performed in patients with a slightly smaller prostate than those used in studies from other countries, and significantly fewer implants were used $(2.24 \pm 0.58)$ than in other studies (3.7-4.9) [18-22]. Despite these differences, in this study, the total IPSS, residual urine, and peak flow showed significant improvement with the small number of PUL implants, and the improvements held after one year.

In TURP and laser vaporization, the incidence of early urge incontinence is $30 \%$ to $40 \%$ [23]; this complication causes many patients to suffer in the early stages of surgery. This study analyzed the IPSS voiding and storage symptom scores separately to assess the initial complications with PUL. The results showed that the voiding symptoms improved and the storage symptoms did not deteriorate in the short and long term. The short-term outcomes are believed to be good because the tissue debris takes a long time to discharge and there is no thermal injury. In addition, no long-term TURP and laser vaporization complications such as TUR syndrome, bladder neck stenosis, urethral stricture and retrograde ejaculation, were observed [23].

The reasons for why ejaculation and erectile dysfunction do not occur with PUL are still unclear. In TURP, retrograde ejaculation occurs in a large number of patients, but some researchers have published data on reducing this complication $[24,25]$. The results from this study suggest the avoidance of thermal injury and the preservation of musculus ejaculatorius and verumontanum for ejaculation and the bladder neck for antegrade ejaculation. PUL is believed to preserve the ejaculatory function because it does not damage the bladder neck or cause thermal injury to the surrounding verumontanum tissue.

The rate of infection after TURP varies from 3.5\% to $21.6 \%$. The known risk factors for infection are preoperative bacteriuria, duration of the procedure, and preoperative hospital stay. Compared to TURP, PUL has strength for the duration of the procedure and preoperative hospital stay. In addition, the advantage that PUL causes no thermal injury and residual necrotic tissue to the prostate appears to result in a lower risk of infection [23].

This study had several limitations. First, retrospective medical data were used and the study did not proceed prospectively. Second, this was a single-arm study. To overcome these limitations, a prospective study comparing PUL and TURP or laser vaporization in small-sized BPH is planned. Third, the ejaculation problems could only be analyzed as yes or no. A future study will include a questionnaire related to erection or ejaculation, such as the International Index of Erectile Function (IIEF), Sexual Health Inventory for Men (SHIM), and The Male Sexual Health 
Questionnaire for Ejaculatory Dysfunction (MSHQ-EjD). Fourth, this study had a small number of one-year follow up patients. Therefore, selection bias may have occurred and the result has a lack of statistical significance. Hence, a long-term follow up study with additional patients is planned to consolidate the statistical significance. Finally, it is burdensome to use multiple implants because of their high cost. As a result, more data from small prostates were used than in other studies.

The correct location of PUL is still unclear because of its higher cost in Korea than in other countries. Therefore, surgeries attempt to avoid procedures that require more than two implants as much as possible. In the present study, however, good results for the total IPSS, residual urine, and peak flow were obtained with an average of only 2.28 implants. If PUL is well screened in smaller prostates, a small number of implants will give good results.

\section{CONCLUSIONS}

PUL is a new type of minimally invasive surgery that has unique advantages. Therefore, it has received considerable attention in the BPH treatment market. In particular, PUL can be performed under local anesthesia, requires short catheterization, has few postoperative complications, and rarely causes ejaculation and erection problems. A retrospective study was performed using smaller implants than those used in other studies because of Korea's special medical environment. Furthermore, the results are expected to be good in appropriately selected patients who want to preserve the ejaculatory function, have a smaller prostate, and refuse general anesthesia.

\section{CONFLICT OF INTEREST}

No potential conflict of interest relevant to this article was reported.

\section{ACKNOWLEDGMENTS}

This study was supported by the Bio \& Medical Technology Development Program of the National Research Foundation (NRF) funded by the Ministry of Science \& Information \& Communication Technology (2018M3A9E8020861).

\section{AUTHOR CONTRIBUTIONS}

J.Y., S.K., and H.C.J. participated in data collection, performed the statistical analysis and wrote the manuscript. D.S., J.S., S.C., and S.W.C. participated in the study design. W.J.B. and S.W.K. participated in the study design and coordination and helped to draft the manuscript. All authors read and approved the final manuscript.

\section{ORCID}

Jonghyup Yang, https://orcid.org/0000-0001-8122-3377 Dongho Shin, https://orcid.org/0000-0001-8496-8825 Jaewoo Sung, https://orcid.org/0000-0002-9106-9788 Shinjay Cho, https://orcid.org/0000-0002-0480-4386

Sungmin Kang, https://orcid.org/0000-0003-1299-8499 Hyun Cheol Jeong, https://orcid.org/0000-0001-8846-1351 Sae Woong Choi, https://orcid.org/0000-0002-3829-939X Woong Jin Bae, https://orcid.org/0000-0001-7703-1161 Sae Woong Kim, https://orcid.org/0000-0002-9127-9400

\section{REFERENCES}

1. Verhamme KM, Dieleman JP, Bleumink GS, Bosch JL, Stricker $\mathrm{BH}$, Sturkenboom MC. Treatment strategies, patterns of drug use and treatment discontinuation in men with LUTS suggestive of benign prostatic hyperplasia: the Triumph project. Eur Urol 2003;44:539-45.

2. Roehrborn CG. Current medical therapies for men with lower urinary tract symptoms and benign prostatic hyperplasia: achievements and limitations. Rev Urol 2008;10:14-25.

3. Park HK, Park H, Cho SY, Bae J, Jeong SJ, Hong SK, et al. The prevalence of benign prostatic hyperplasia in elderly men in Korea: a community-based study. Korean J Urol 2009;50: 843-7.

4. AUA Practice Guidelines Committee. AUA guideline on management of benign prostatic hyperplasia (2003). Chapter 1 : diagnosis and treatment recommendations. J Urol 2003;170: 530-47.

5. Nickel JC, Saad F. The American Urological Association 2003 guideline on management of benign prostatic hyperplasia: a Canadian opinion. Can J Urol 2004;11:2186-93.

6. McVary KT, Roehrborn CG, Avins AL, Barry MJ, Bruskewitz RC, Donnell RF, et al. Update on AUA guideline on the management of benign prostatic hyperplasia. J Urol 2011;185:1793-803.

7. Bouza C, López T, Magro A, Navalpotro L, Amate JM. Systematic review and meta-analysis of transurethral needle ablation in symptomatic benign prostatic hyperplasia. BMC Urol 2006;6: 
14.

8. Andersen $\mathrm{M}$, Walter $\mathrm{S}$. [Microwave thermotherapy for benign prostatic hyperplasia. A survey of a Cochrane review]. Ugeskr Laeger 2009;171:2281-4. Danish.

9. Chin PT, Bolton DM, Jack G, Rashid P, Thavaseelan J, Yu RJ, et al. Prostatic urethral lift: two-year results after treatment for lower urinary tract symptoms secondary to benign prostatic hyperplasia. Urology 2012;79:5-11.

10. McNicholas TA, Woo HH, Chin PT, Bolton D, Fernández Arjona $M$, Sievert KD, et al. Minimally invasive prostatic urethral lift: surgical technique and multinational experience. Eur Urol 2013; 64:292-9.

11. Jones $P$, Rai BP, Aboumarzouk OM, Somani BK. Urolift: a new chapter in benign prostate hyperplasia (BPH) therapy. Ann Transl Med 2016;4:116.

12. Jones $P$, Rajkumar $G N$, Rai BP, Aboumarzouk OM, Cleaveland $P$, Srirangam SJ, et al. Medium-term outcomes of urolift (minimum 12 months follow-up): evidence from a systematic review. Urology 2016;97:20-4.

13. Statistics Korea. Korean Statistical Information Service [Internet]. Daejeon: Statistics Korea; 2014 [cited 2018 Oct 5]. Available from: http://kosis.kr/index/index.do.

14. Lee YJ, Lee JW, Park J, Seo SI, Chung JI, Yoo TK, et al. Nationwide incidence and treatment pattern of benign prostatic hyperplasia in Korea. Investig Clin Urol 2016;57:424-30.

15. Barrack ER, Bujnovszky P, Walsh PC. Subcellular distribution of androgen receptors in human normal, benign hyperplastic, and malignant prostatic tissues: characterization of nuclear saltresistant receptors. Cancer Res 1983;43:1107-16.

16. Martin DJ, Mulhall JP. Enlarging the scope of managing benign prostatic hyperplasia: addressing sexual function and quality of life. Int J Clin Pract 2005;59:579-90.

17. Ulchaker JC, Martinson MS. Cost-effectiveness analysis of six therapies for the treatment of lower urinary tract symptoms due to benign prostatic hyperplasia. Clinicoecon Outcomes Res
2017; 10:29-43.

18. Roehrborn CG, Gange SN, Shore ND, Giddens JL, Bolton DM, Cowan $\mathrm{BE}$, et al. The prostatic urethral lift for the treatment of lower urinary tract symptoms associated with prostate enlargement due to benign prostatic hyperplasia: the L.I.F.T. Study. J Urol 2013;190:2161-7.

19. Cantwell AL, Bogache WK, Richardson SF, Tutrone RF, Barkin J, Fagelson JE, et al. Multicentre prospective crossover study of the 'prostatic urethral lift' for the treatment of lower urinary tract symptoms secondary to benign prostatic hyperplasia. BJU Int 2014;113:615-22.

20. Shore N, Freedman S, Gange S, Moseley W, Heron S, Tutrone R, et al. Prospective multi-center study elucidating patient experience after prostatic urethral lift. Can J Urol 2014;21:7094-101.

21. Rukstalis DB. Prostatic urethral lift: a novel approach for managing symptomatic BPH in the aging man. Can J Urol 2015;22 Suppl 1:67-74.

22. Sønksen J, Barber NJ, Speakman MJ, Berges R, Wetterauer U, Greene D, et al. Prospective, randomized, multinational study of prostatic urethral lift versus transurethral resection of the prostate: 12-month results from the BPH6 study. Eur Urol 2015;68:643-52.

23. Rassweiler J, Teber D, Kuntz R, Hofmann R. Complications of transurethral resection of the prostate (TURP)--incidence, management, and prevention. Eur Urol 2006;50:969-79; discussion 980.

24. Alloussi SH, Lang C, Eichel R, Alloussi S. Ejaculation-preserving transurethral resection of prostate and bladder neck: short- and long-term results of a new innovative resection technique. J Endourol 2014;28:84-9.

25. Schoenthaler M, Sievert KD, Schoeb DS, Miernik A, Kunit T, Hein S, et al. Combined prostatic urethral lift and remodeling of the prostate and bladder neck: a modified transurethral approach in the treatment of symptomatic lower urinary tract obstruction. World J Urol 2018;36:1111-6. 